\title{
AUTOMATA-BASED REPRESENTATIONS FOR INFINITE GRAPHS *
}

\author{
SAlvatore LA TORRE $^{1}$ AND MARgherita NAPOLI ${ }^{1}$
}

\begin{abstract}
New compact representations of infinite graphs are investigated. Finite automata are used to represent labelled hypergraphs which can be also multi-graphs. Our approach consists of a general framework where vertices are represented by a regular prefixfree language and edges are represented by a regular language and a function over tuples. We consider three different functions over tuples: given a tuple the first function returns its first difference, the second one returns its suffix and the last one returns its infixes. The first-difference function is substantially a direct generalization to infinite multi-hypergraphs of the representation introduced by Ehrenfeucht et al. for finite graphs. This representation, though very interesting for finite graphs, turns out to be quite unsatisfactory for infinite graphs. The other two functions we consider while preserving some interesting features of their representation also achieves a high expressive power. As a matter of fact, our formalism either with the suffix or infix function results to be more powerful than the equational graphs introduced by Courcelle and the simple graphs defined by Caucal. The monadic second order theories of these two classes of graphs are undecidable, but still many interesting graph properties are decidable. The use of a regular prefixfree language to represent the vertices allows (fixed the language of the edges) to express a graph by a labelled tree, moreover, the use of finite automata to represent the edges allows the verification of graph properties.
\end{abstract}

Mathematics Subject Classification. 05C62.

\footnotetext{
Keywords and phrases: Infinite Graphs, Formal Languages.

* Partially supported by the M.U.R.S.T. in the framework of the projects "Tecniche formali per la specifica, l'analisi, la verifica, la sintesi e la trasformazione di sistemi software" and "Theory of Concurrency, Higher Order and Types".

1 Dipartimento di Informatica ed Applicazioni, Università degli Studi di Salerno, Baronissi, Italia; e-mail: $\{$ sallat, napoli\}@dia.unisa.it
} 


\section{INTRODUCTION}

Infinite graphs have been intensively used for modelling non-terminating concurrent programs and, often, turn out to be very useful in studying properties of large graphs. In this context the use of a suitable graph representation has a relevant significance in terms of efficient computations and expressiveness. Succinctness, expressiveness and decidability of the main graph properties are goals to pursue in a graph representation. In the last decade, many efforts have been done to obtain small specifications of graphs. A well supported idea has been that of representing graphs by expressions or trees $[1,6,15]$.

In [3], Bauderon et al. define finite hyper-graphs in a compositional way, that is they use graph expressions built from basic ones by applications of simple graph operations corresponding to hyper-edge replacements. Systems of graph equations with this kind of expressions provide a way to define infinite hyper-graphs, called equational graphs [7]. In the recent paper [2], the class of simple equational hypergraphs is extended by allowing vertex replacement. This way it is obtained a characterization of the class of simple graphs whose monadic second-order theory is decidable and that strictly contains the simple equational graphs [5].

Another way to specify finite graphs, recently introduced by Ehrenfeucht et al. [9], relies on finite prefix-free languages of strings whose alphabets have themselves a graph structure. The alphabet is a loop-free finite graph and the vertices are words from a finite prefix-free language. There is an edge between two vertices if and only if the pair of the first two symbols, at which the two corresponding strings differ (first difference), is an edge of the alphabet graph. In this paper we introduce a new way for specifying infinite hyper-graphs via regular languages. Our approach consists of a general framework where vertices are represented by a regular prefixfree language as in [9] and edges are represented by a regular language $P$ and a function $f$ over tuples with the meaning that the considered graph has an edge linking the ordered sequence of vertices $x_{1}, \ldots, x_{k}$ if $f\left(x_{1}, \ldots, x_{k}\right) \cap P \neq \emptyset$. We consider three different functions over tuples: first, suffix and infix. For a tuple $x_{1}, \ldots, x_{k}$, first $\left(x_{1}, \ldots, x_{k}\right)$ gives the first difference that occurs in $x_{1}, \ldots, x_{k}$, that is the tuple of first symbols of the words obtained from $x_{1}, \ldots, x_{k}$ cutting their common longest prefix, if there exist $i$ and $j$ such that $x_{i} \neq x_{j}$. In the other case, first $\left(x_{1}, \ldots, x_{k}\right)=\left\{\left(x_{1}, \ldots, x_{k}\right)\right\}$. In an analogous way, the function $\operatorname{suffix}\left(x_{1}, \ldots, x_{k}\right)$ gives the suffix of $\left(x_{1}, \ldots, x_{k}\right)$, that is the tuple obtained from $x_{1}, \ldots, x_{k}$ cutting their common longest prefix, and $\operatorname{infix}\left(x_{1}, \ldots, x_{k}\right)$ gives the infixes of $\left(x_{1}, \ldots, x_{k}\right)$, that is the prefixes of the words obtained from $x_{1}, \ldots, x_{k}$ by cutting their longest common prefix. Our graph representation with the function first is substantially a direct generalization to infinite multi-hyper-graphs of the representation introduced in [9] for finite graphs. This representation, though very interesting for finite graphs, turns out to be inadequate when the goal is the representation of infinite graphs. The main reason of this is that, among the infinite graphs, we are able only to represent graphs with an infinite degree or that are the union of infinitely many maximally connected subgraphs of finite 
size. The other two functions we consider provide a higher expressive power to our representation. The suffix-based representation (suffix-representable graphs) is less expressive than the one based on the infixes (infix-representable graphs) relatively to labelled graphs while it is equivalent to this last on the unlabelled graphs. Other advantages in using the infixes are a more concise representation and a higher expressive power in the case of a finite $P$ for both labelled and unlabelled graphs.

Our approach preserves the finite representation of the (possibly infinite) graphs and allows to specify a meaningful class of infinite hyper-graphs. As a matter of fact, both the classes of suffix-representable graphs and infix-representable graphs result to be strictly larger than the class of equational graphs [7] and the class of simple graphs introduced in [5]. In a similar way as in [9], the use of regular languages allows us to inherit concepts and ideas from the formal language theory and use them for graphs. In particular we are interested in the relationships between language operations and graph operations: the relevance of this investigation is due to the possibility of performing graph transformations by manipulating the regular languages used for the graph representation. The main classes of graphs we introduce have an undecidable monadic second order theory but it is still possible for them to answer some typical graph questions, which may be difficult when infinite graphs are considered, such as the existence of a sink, a source, a transient or an isolated vertex, and to determine for example the degree of a vertex. Moreover, the use of a regular prefix-free language to represent the vertices allows (fixed the language of the edges) to express a graph by a labelled tree.

In Section 2 we introduce some notation and formally define our representation. The suffix-representable graphs are compared to the equational graphs in Section 3 and to the simple graphs introduced by Caucal in Section 4. In Section 5 the class of infix-representable graphs is compared to the class of suffix-representable graphs and some graph properties and algorithms are studied. Finally, in Section 6, we give few conclusions and address some future directions of this research.

\section{Graph Representation over a Regular language OF TUPLES}

In this section we introduce a new framework to represent multi-hyper-graphs. We start giving some basic definitions and introducing the notation we will use throughout this paper. We suppose that the reader is familiar with the basic concepts of formal languages (see for example [12]) and only recall that $L$ is said prefix-free if for every distinct $x, y \in L$ it holds that $x$ is not a prefix of $y$. In the following we will use $N$ to denote the set of the non-negative integers. A multiset $m s$ over a finite set $\Delta$ is a mapping from $\Delta$ into $N \cup\{\infty\}$ and $m s(a)$ is said the multiplicity of $a$, for $a \in \Delta$. The set of the multisets over $\Delta$ is denoted by $M S(\Delta)$ and the multiset mapping $a$ into 0 for any $a \in \Delta$ is denoted by 0 . Given two multisets $m s$ and $m s^{\prime}$, we say that $m s \leq m s^{\prime}$ if $m s(a) \leq m s^{\prime}(a)$ for every $a \in \Delta$ and we denote with $m s+m s^{\prime}$ the multiset mapping each $a \in \Delta$ into 
$m s(a)+m s^{\prime}(a)$. Moreover, for each $a \in \Delta$, with $m s-m s^{\prime}$ we denote the multiset mapping $a$ into $m s(a)-m s^{\prime}(a)$, if $m s(a)-m s^{\prime}(a) \geq 0$ and $m s^{\prime}(a)<\infty$, and 0 , otherwise. Obviously, $\infty+k=k+\infty=\infty+\infty=\infty-k=\infty$ holds. Sometimes in the following we denote the multisets which are also sets with the usual set notation.

In this paper we deal with directed labelled hyper-graphs which can be also multi-graphs, that is they can have many hyper-edges linking any ordered tuple of vertices. We consider a directed labelled hyper-edge as given by a sequence of vertices $\left(v_{1}, \ldots, v_{k}\right)$ and a multiset $m s$, with the meaning that there are exactly $m s(a)$ directed hyper-edges linking $v_{1}, \ldots, v_{k}$ and labelled by $a \in \Delta$. We denote each of them with $\left(\left(v_{1}, \ldots, v_{k}\right), a\right)$ and we say that a hyper-edge is incident to each of the vertices $v_{1}, \ldots, v_{k}$ that it links. Note that we are not interested in distinguishing among hyper-edges linking the same tuple of vertices and having the same label. Labels are taken from a ranked alphabet, that is a pair $(\Delta, \tau)$ where $\Delta$ is an alphabet and $\tau$ is a mapping from $\Delta$ into the set of positive integers.

Definition 2.1. Let $(\Delta, \tau)$ be a ranked alphabet. A labelled hyper-graph is a pair $G=(V, l a b)$ where:

- $V$ is the set of the vertices;

- lab : $\bigcup_{k=1}^{\infty} V^{k} \rightarrow M S(\Delta)$ is a total mapping, called labelling function, such that, for every $a \in \Delta$ and $v_{1}, \ldots, v_{k} \in V, \operatorname{lab}\left(v_{1}, \ldots, v_{k}\right)(a)>0$ implies $\tau(a)=k$.

A tuple $\left(v_{1}, \ldots, v_{k}\right) \in V^{k}$ is called a hyper-edge of $G$ of length $k$ if $l a b\left(v_{1}, \ldots, v_{k}\right) \neq \mathbf{0}$. Note that the length of a hyper-edge is upperbounded by $\tau(\Delta)=\max \{\tau(a) / a \in \Delta\}$. From now on, if it is not differently stated we will use the word graph for a labelled hyper-graph and its hyper-edges are simply called edges. Moreover, we will often denote with $E_{G}=\left\{\left(v_{1}, \ldots, v_{k}\right) \in V^{k} / k \geq\right.$ $\left.1, \operatorname{lab}\left(v_{1}, \ldots, v_{k}\right) \neq \mathbf{0}\right\}$ the set of the hyper-edges of $G$. In Section 4 we will consider simple graphs, that is graphs whose labelling function gives mappings from $\Delta$ into $\{0,1\}$. A subgraph of a graph $G=(V, l a b)$ is a graph $G^{\prime}=\left(V^{\prime}, l a b^{\prime}\right)$ such that $V^{\prime} \subseteq V$ and $l a b^{\prime}\left(v_{1}, \ldots, v_{k}\right) \leq l a b\left(v_{1}, \ldots, v_{k}\right)$ for each $v_{1}, \ldots, v_{k} \in V^{\prime}$. In this case we say that $G^{\prime} \subseteq G$. If $l a b^{\prime}\left(v_{1}, \ldots, v_{k}\right)=\operatorname{lab}\left(v_{1}, \ldots, v_{k}\right)$ for all $v_{1}, \ldots, v_{k} \in V^{\prime}$, then $G^{\prime}$ is said to be the subgraph of $G$ induced by $V^{\prime}$ and we denote it by $G_{\mid V^{\prime}}$. Let $G=(V, l a b)$ be a graph and $\approx$ be an equivalence relation on $V$. We define the quotient graph, denoted by $G_{\approx}$, the graph $\left(V_{\approx}, l a b \approx\right)$ where: $V_{\approx}=\{[v] / v \in V\}$ and $l a b \approx\left(\left[v_{1}\right], \ldots,\left[v_{k}\right]\right)=\sum_{v_{i}^{\prime} \in\left[v_{i}\right]} l a b\left(v_{1}, \ldots, v_{k}\right)$. Let $G_{i}=\left(V_{i}, l a b_{i}\right)$ for $i=1,2$ be graphs, an isomorphism between graphs $\phi: V_{1} \rightarrow V_{2}$ is a bijective mapping such that: $l a b_{1}\left(v_{1}, \ldots, v_{k}\right)=l a b_{2}\left(\phi\left(v_{1}\right), \ldots, \phi\left(v_{k}\right)\right)$. Finally, we define the limit of a succession of finite graphs. Let $\left\{G_{n}\right\}_{n>0}$ be a succession of finite graphs, we say that a (possibly infinite) graph $G$, denoted by $\lim _{n} G_{n}$, is the limit for $n \rightarrow \infty$ of the succession $\left\{G_{n}\right\}_{n>0}$ if $G_{n} \subseteq G$ for all $n>0$ and for all finite subsets of vertices $V$ of $G$ there exists an $n$ such that $G_{\mid V} \subseteq G_{n}$. A succession of finite graphs $\left\{G_{n}\right\}_{n>0}$ such that $G_{n}=\left(V_{n}, l a b_{n}\right)$ and $G_{n} \subseteq G_{n+1}$ is said to be a monotonic succession of graphs and we have that $\lim _{n} G_{n}=\left(\bigcup_{n>0} V_{n}, \lim _{n} l a b_{n}\right)$. Note that 
$\left\{l a b_{n}\right\}_{n>0}$ is a monotonic succession of functions, thus its limit always exists. The following result holds.

Proposition 2.2. Given two monotonic successions of finite graphs $\left\{G_{n}\right\}_{n>0}$ and $\left\{G_{n}^{\prime}\right\}_{n>0}$ such that $G_{n}$ is isomorphic to $G_{n}^{\prime}$ for $n>0$, then $\lim _{n} G_{n}$ is isomorphic to $\lim _{n} G_{n}^{\prime}$.

Proof. Directly from the definition of limit of a monotonic succession of graphs.

In the rest of this section we introduce a new way of representing graphs. We represent the vertices by a prefix-free regular language and the edges by a language recognized by a synchronized finite automaton (parallel regularity) with multiple tapes along with a function over tuples. The synchronized automaton we consider is a simplified version of the $r$-tape one-way acceptors introduced by Rabin and Scott (see [11] for a survey). A synchronized finite automaton with multiple heads and multiple tapes is used to accept languages of tuples of words: the length of each tuple is less than or equal to the number of the tapes. Each head has its own tape and moves leftwards at each computational step to read the next input symbol until the end of the word on its tape is reached. All the heads which have not reached yet the end of the words on their tape move synchronously. More formally, we have the following definition:

Definition 2.3. Let $r$ be a positive integer number. A synchronized $r$-tape finite automaton, denoted by $r$-FA, is a tuple $\left(Q, \Sigma, \downarrow, q_{0}, \Delta, F\right)$ where:

- $Q$ is a finite set of states;

- $\Sigma$ is an alphabet;

- $\emptyset \notin \Sigma$ is the blank symbol;

- $q_{0}$ is the initial state;

- $F \subseteq Q$ is the set of final states;

- $\Delta \subseteq \bigcup_{i=1}^{r} Q \times(\Sigma \cup\{\emptyset\})^{i} \times Q$ is the transition relation.

A $k$-tuple $\left(x_{1}, \ldots, x_{k}\right) \in \Sigma^{k}$ is accepted by an $r$-FA $A$ if $k \leq r$ and $A$ enters a final state after reading the $k$-tuple $\left(x_{1} \natural^{m-\left|x_{1}\right|}, \ldots, x_{k}^{m-\left|x_{k}\right|}\right)$ where $m=$ $\max \left\{\left|x_{1}\right|, \ldots,\left|x_{k}\right|\right\}$. The language accepted by $A$, denoted by $L(A)$, is said to be regular in parallel. Note that all the results concerning the closure properties and the decidability holding for the finite automaton still hold for the class of $r$-FA.

We represent a graph with a prefix-free language (the vertices), a function $f$ over the tuples of vertices, a set of tuples $P$ and a labelling function with the meaning that there is an edge with vertices $x_{1}, \ldots, x_{k}$ if $f\left(x_{1}, \ldots, x_{k}\right)$ has a non-empty intersection with $P$ and the labelling function maps one of the tuples belonging to this intersection into a multiset which differs from $\mathbf{0}$.

Definition 2.4. Let $\Sigma$ be an alphabet, $(\Delta, \tau)$ be a ranked alphabet, $f$ be a mapping from $\bigcup_{j=1}^{\infty}\left(\Sigma^{+}\right)^{j}$ into the subsets of $\bigcup_{j=1}^{\infty}\left(\Sigma^{+}\right)^{j}$ which preserves the arity of tuples (i.e., $\left.f\left(x_{1}, \ldots, x_{k}\right) \subseteq\left(\Sigma^{+}\right)^{k}\right), L \subseteq \Sigma^{+}$be a regular prefix-free language, $P \subseteq \bigcup_{j=1}^{\infty}\left(\Sigma^{+}\right)^{j}$ be such that $P=\bigcup_{i=1}^{m} P_{i}$ where $P_{i}$ is regular in parallel for all $i=1, \ldots, m$ and $l a b$ be a total mapping from $P$ into $M S(\Delta)$ such that 
$\left|l a b\left(P_{i}\right)\right|=1$ for all $i=1, \ldots, m$. We denote with $\operatorname{gra}(L, f, P, l a b)$ the graph $G=\left(L, l a b^{\prime}\right)$ where $l a b^{\prime}: \bigcup_{k=1}^{\infty} L^{k} \rightarrow M S(\Delta)$ is such that:

$$
l a b^{\prime}\left(x_{1}, \ldots, x_{k}\right)=\sum_{\left(y_{1}, \ldots, y_{k}\right) \in f\left(x_{1}, \ldots, x_{k}\right) \cap P} l a b\left(y_{1}, \ldots, y_{k}\right) .
$$

We say that the graph $G$ is $f$-representable.

The function $f$ can be defined in several ways. For example, consider the function first $\left(x_{1}, \ldots, x_{k}\right)$ which gives the first difference occurring in $x_{1}, \ldots, x_{k}$, that is the set containing the tuple of first symbols of the words obtained from $x_{1}, \ldots, x_{k} \in L$ cutting their common longest prefix, if there exist $i$ and $j$ such that $x_{i} \neq x_{j}$, and first $\left(x_{1}, \ldots, x_{k}\right)=\left\{\left(x_{1}, \ldots, x_{k}\right)\right\}$, otherwise. Our graph representation with the function first is the obvious extension to infinite graphs of the graph representation introduced in [9]. It is easy to verify that any infinite graph which first-representable has either infinite degree or is the disjoint union of infinitely many maximally connected subgraphs of finite size, thus there are many interesting infinite graphs that are not captured by this representation.

In the rest of the paper we will focus on two classes of $f$-representable graphs obtained respectively via the functions suffix and infix. Functions suffix and infix give respectively the suffix and the infixes of a tuple. Intuitively, by suffix of a tuple $\left(x_{1}, \ldots, x_{k}\right)$ we mean the tuple obtained from $x_{1}, \ldots, x_{k}$ by cutting their longest common prefix, if there exists $i$ and $j$ such that $x_{i} \neq x_{j}$, and the tuple itself, otherwise. This last case is important since allows us to represent self-loops in graphs.

Definition 2.5. Let $\Sigma$ be an alphabet. For any $x_{1}, \ldots, x_{k} \in \Sigma^{+}$we define suffix $\left(x_{1}, \ldots, x_{k}\right)$ as the set containing either the $k$-tuple $\left(a_{1} y_{1}, \ldots, a_{k} y_{k}\right)$, if $x_{i}=$ $x a_{i} y_{i}, a_{i} \in \Sigma$ and $\exists j, m$ such that $a_{j} \neq a_{m}$, or the $k$-tuple $\left(x_{1}, \ldots, x_{k}\right)$, otherwise.

Analogously, by infixes of a tuple $\left(x_{1}, \ldots, x_{k}\right)$ we mean the tuples obtained from $x_{1}, \ldots, x_{k}$ by cutting their longest common prefix and ignoring an arbitrary suffix, if there exists $i$ and $j$ such that $x_{i} \neq x_{j}$, and the tuple of the infixes of the $x_{j}$, otherwise.

Definition 2.6. Let $\Sigma$ be an alphabet. For any $x_{1}, \ldots, x_{k} \in \Sigma^{+}$we define $\operatorname{infix}\left(x_{1}, \ldots, x_{k}\right)$ as the set of $k$-tuple $\left(a_{1} y_{1}, \ldots, a_{k} y_{k}\right)$, if $x_{i}=x a_{i} y_{i} x_{i}^{\prime}, a_{i} \in \Sigma$ and $\exists j, m$ such that $a_{j} \neq a_{m}$, and as the set containing all the $k$-tuples $(x, \ldots, x)$ such that $x$ is an infix of $x_{j}$, otherwise.

We will compare suffix-representable graphs to infix-representable graphs in Section 5.1. The following example gives a connected infinite graph with finite degree which is both suffix and infix-representable. As a consequence, both the classes of suffix and infix-representable graphs strictly contain the class of firstrepresentable graphs.

Example 2.7. Let $G=(N, l a b)$ be such that:

1. $E_{G}=\{(2 i+1,2 i+3) / i \in N\} \cup\{(2 i+1,2 i) / i \in N\} \cup\{(2 i+2,2 i) / i \in N\}$;

2. $l a b(2 i+1,2 i+3)=\{a\}, \operatorname{lab}(2 i+1,2 i)=\{b\}$ and $\operatorname{lab}(2 i+2,2 i)=\{c\}$. 
We show that $G$ is suffix-representable and thus infix-representable. In fact, consider the graph $G^{\prime}=\operatorname{gra}\left(v+y+1^{*}(w+z)\right.$, suffix, $\{(v, y),(v, w),(w, z),(z, y),(w, 1 w)$, $\left.(1 z, z)\}, l a b^{\prime}\right)$ where $l a b^{\prime}(v, y)=l a b^{\prime}(w, z)=\{b\}, l a b^{\prime}(v, w)=l a b^{\prime}(w, 1 w)=\{a\}$ and $l a b^{\prime}(z, y)=l a b^{\prime}(1 z, z)=\{c\}$ (see Fig. 1). Then, it easy to see that $G^{\prime}$ is isomorphic to $G$.

It is worth noticing that the graph $G^{\prime}$ from the above example is also isomorphic to $\operatorname{gra}\left(v+y+1^{*}(w+z)\right.$, infix, $\left.\{(v, y),(v, w),(w, z),(z, y),(w, 1 w),(1 z, z)\}, l a b^{\prime}\right)$, while in general $\operatorname{gra}(L$, infix, $P, l a b)$ and $\operatorname{gra}(L$, suffix, $P, l a b)$ are not isomorphic graphs.

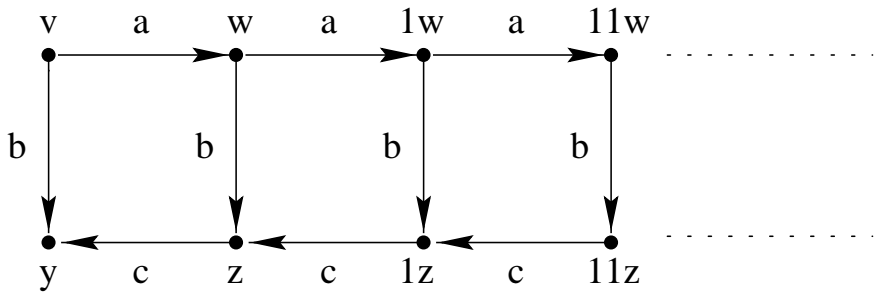

Figure 1. A graphical representation of $G^{\prime}$.

We end this section with two interesting properties of $f$-representable graphs. The first one is related to the continuity of the function gra.

Proposition 2.8. Let $\left\{\operatorname{gra}\left(L_{n}, f, P_{n}, l a b_{n}\right)\right\}_{n>0}$ be a succession of finite graphs such that $L_{n} \subseteq L_{n+1}, P_{n} \subseteq P_{n+1}$ and $l a b_{n} \leq l a b_{n+1}$. Then, it holds that $\lim _{n} \operatorname{gra}\left(L_{n}, f, P_{n}, l a b_{n}\right)=\operatorname{gra}\left(\bigcup_{n>0} L_{n}, f, \bigcup_{n>0} P_{n}, \lim _{n} l a b_{n}\right)$.

Proof. The above statement is a direct consequence of the fact that the succession of finite graphs $\left\{\operatorname{gra}\left(L_{n}, f, P_{n}, l a b_{n}\right)\right\}_{n>0}$ is monotonic.

The second property gives a normal form for $f$-representable graphs for $f \in$ \{first, suffix, infix .

Proposition 2.9. Given an $f$-representable graph $G$ for $f \in\{$ first, suffix, infix $\}$, there exists an $f$-representable graph gra $\left(L, f, \bigcup_{i=1}^{m} P_{i}, l a b\right)$ which is isomorphic to $G$ and such that for $i=1, \ldots, m$ either:

- for every $\left(x_{1}, \ldots, x_{k}\right) \in P_{i}$ there is not a $y \in \Sigma^{+}$such that $x_{j}=y x_{j}^{\prime}$ for $j=1, \ldots, k$ or

- for every $\left(x_{1}, \ldots, x_{k}\right) \in P_{i}, x_{1}=\ldots=x_{k}$.

Proof. Directly from the definitions and the properties of $r$-FAs.

\section{EQUATIONAL GRAPHS vs. SUFFIX-REPRESENTABLE GRAPHS}

In this section, we briefly recall the notion of the equational graphs as defined in [7], then we compare their expressive power to our representation and give a 
way to obtain an automata-based description of an equational graph. At this aim we start recalling the definition of an $n$-graph and some graph operations.

An $n$-graph is a tuple $G=(V, E, l a b, s r c)$ where $(V, l a b)$ is a graph with set of hyper-edges $E$, said the graph of $G$, and $\operatorname{src}$ is a total mapping from $\{1, \ldots, n\}$ into $V$. For the $n$-graphs we will use some definitions given for graphs, and in particular the quotient of an $n$-graph and the notion of subgraph are defined in an analogous way as for graphs.

Let $G=(V, E, l a b, s r c)$ and $G^{\prime}=\left(V^{\prime}, E^{\prime}, l a b^{\prime}, s r c^{\prime}\right)$ be respectively an $n$-graph and an $n^{\prime}$-graph such that $V \cap V^{\prime}=\emptyset$ and $E \cap E^{\prime}=\emptyset$. The disjoint union of $G$ and $G^{\prime}$, denoted by $G \oplus G^{\prime}$, is defined as the $\left(n+n^{\prime}\right)$-graph $\left(V \cup V^{\prime}, E \cup E^{\prime}, l a b \cup l a b^{\prime}, s r c^{\prime \prime}\right)$ where $s r c^{\prime \prime}$ is the mapping defining the sequence which is the concatenation of the sequences defined by $\operatorname{src}$ and $s r c^{\prime}$. Moreover, let $f:\{1, \ldots, m\} \rightarrow\{1, \ldots, n\}$ be a total mapping, the $s r c$-redefinition map $\sigma_{f}$ is defined as $\sigma_{f}(G)=(V, E, l a b, \operatorname{src} \circ f)$ where $\circ$ is the usual composition of functions. Let $\delta$ be an equivalence relation over $\{1, \ldots, n\}$, the $s r c$-fusion map $\theta_{\delta}$ is defined as $\theta_{\delta}(G)=G \approx$, where $G \approx$ is the quotient with respect to the equivalence relation on $V$ defined as:

$$
v \approx v^{\prime} \Leftrightarrow v=v^{\prime} \text { or }\left(v=\operatorname{src}(i), v^{\prime}=\operatorname{src}(j) \text { and }(i, j) \in \delta\right) .
$$

Finally, let $(\Delta, \tau)$ be a ranked alphabet, $\left(v_{1}, \ldots, v_{n^{\prime}}\right)$ be a tuple belonging to $E$ and $a \in \Delta$ be such that $l a b\left(v_{1}, \ldots, v_{n^{\prime}}\right)(a) \neq \mathbf{0}$. The n-graph $G_{\approx}^{\prime \prime}$, which is obtained by substituting $G^{\prime}$ for an edge $\left(\left(v_{1}, \ldots, v_{n^{\prime}}\right), a\right)$ in $G$ and is denoted as $G\left[\left(\left(v_{1}, \ldots, v_{n^{\prime}}\right), a\right) \leftarrow G^{\prime}\right]$, is given by

- $G^{\prime \prime}=\sigma_{f}\left(\bar{G} \oplus G^{\prime}\right)$ with $f:\{1, \ldots, n\} \rightarrow\left\{1, \ldots, n+n^{\prime}\right\}$ defined as $f(i)=i$ for $i=1, \ldots, n$ and $\bar{G}=(V, E, \overline{l a b}, s r c)$ where $\overline{l a b}\left(w_{1}, \ldots, w_{k}\right)=l a b\left(w_{1}, \ldots, w_{k}\right)$ for $\left(w_{1}, \ldots, w_{k}\right) \neq\left(v_{1}, \ldots, v_{n^{\prime}}\right)$ and $\overline{l a b}\left(v_{1}, \ldots, v_{n^{\prime}}\right)=l a b\left(v_{1}, \ldots, v_{n^{\prime}}\right)-\{a\}$;

- $\approx$ is an equivalence relation on $V \cup V^{\prime}$ defined as:

$$
v \approx v^{\prime} \Leftrightarrow v=v^{\prime} \text { or }\left(v=v_{i} \text { and } v^{\prime}=\operatorname{src}^{\prime}(i)\right) .
$$

Roughly speaking, the substitution of $G^{\prime}$ for the edge $\left(\left(v_{1}, \ldots, v_{n^{\prime}}\right), a\right)$ in $G$ consists of the deletion in $G$ of an $a$-labelled edge linking the tuple $\left(v_{1}, \ldots, v_{n^{\prime}}\right)$ and the gluing of $G^{\prime}$ and $G$ by the fusion of $v_{i}$ with $\operatorname{src}^{\prime}(i)$, for $i=1, \ldots, n^{\prime}$. Note that the n-graph $\bar{G}$ is the result of the deletion in $G$ of one of the $a$-labelled edges linking $\left(v_{1}, \ldots, v_{n^{\prime}}\right)$.

The above $n$-graph substitution can be generalized by defining a substitution of all the edges which have a given label. Thus, for $i=1, \ldots, m$ let $G_{i}$ be an $n_{i}$-graph and $a_{i} \in \Delta$ be such that $\tau\left(a_{i}\right)=n_{i}$. We denote with $G\left[a_{1} \leftarrow G_{1}, \ldots, a_{m} \leftarrow G_{m}\right]$ the result of simultaneously substituting $G_{i}$ for every edge which is labelled by $a_{i}$ in $G$.

A graph expression of type $n$ is defined from symbols in a ranked alphabet $(\Delta, \tau)$, by means of the operators $\hat{\oplus}, \hat{\theta}_{\delta}$ and $\hat{\sigma}_{f}$ whose meaning is obtained by the meaning of the corresponding operators on n-graphs. In fact, to each graph expression $e$ of type $n$ it is possible to associate a $n$-graph, denoted with $\operatorname{val}(e)$, which represents the evaluation of the expression $e$. By considering some unknowns in the set $\Delta$, graph equations and systems of graph equations are defined in an obvious way. A solution to such a system is a tuple of graph expressions whose depth (i.e. the nesting of the operators) may be infinite. As matter of fact, there 
exists an evaluation mapping which associates a unique (up to an isomorphism) $n$-graph to an infinite graph expression and we denote it again with val. More details can be found in [7].

Let $S$ be the system of graph equations $\left\langle u_{1}=e_{1}, \ldots, u_{m}=e_{m}\right\rangle$ in the unknowns $u_{1}, \ldots, u_{m}$. If $S$ satisfies the Greibach condition (that none of the $e_{i}$ is an unknown), then $S$ has a unique solution $\left(U_{1}, \ldots, U_{m}\right)$ and the graphs of the elements of the $m$-tuple $\left(\operatorname{val}\left(U_{1}\right), \ldots, \operatorname{val}\left(U_{m}\right)\right)$ are said equational graphs [7].

In the following, $S=\left\langle u_{1}=e_{1}, \ldots, u_{m}=e_{m}\right\rangle$ is a system of graph equations in the unknowns $u_{1}, \ldots, u_{m}$ and satisfying the Greibach condition, $\left(U_{1}, \ldots, U_{m}\right)$ is its solution and the $n_{h}$-graphs $\operatorname{val}\left(e_{h}\right)=\left(V_{h}, E_{h}, l a b_{h}, s r c_{h}\right)$ are such that, for all $i \neq j, V_{i} \cap V_{j}=\emptyset$ holds. Without loss of generality we can suppose that for each unknown $u_{i}$ and for each graph expression $e_{j}$, there is at most one occurrence of $u_{i}$ in $e_{j}$. Furthermore, we denote with $\operatorname{unk}\left(e_{j}\right)$ the set of the indexes of the unknowns appearing in $e_{j}$ and we will use the projection functions $\pi_{k}^{h}\left(e_{j}\right)$ to denote the $k$-th vertex of the edge labelled by $u_{h}$ in the graph $\operatorname{val}\left(e_{j}\right)$.

For each $i=1, \ldots, m$ we can define the structure of $U_{i}$ according to the system $S$ as the tree $\operatorname{struct}_{i}^{S}: \operatorname{dom}\left(\right.$ struct $\left._{i}^{S}\right) \rightarrow\left\{e_{1}, \ldots, e_{m}\right\}$ such that:

- $\operatorname{dom}\left(\operatorname{struct} t_{i}^{S}\right) \subseteq\{1, \ldots, m\}^{*}$

- $\epsilon \in \operatorname{dom}\left(\operatorname{struct}_{i}^{S}\right)$ and $\operatorname{struct}_{i}^{S}(\epsilon)=e_{i}$;

- if $x \in \operatorname{dom}\left(\operatorname{struct}_{i}^{S}\right)$ then $\left.\forall k \in \operatorname{unk}\left(\operatorname{struct}_{i}^{S}(x)\right): x k \in \operatorname{dom}_{\text {struct }}^{S}\right)$ and $\operatorname{struct}_{i}^{S}(x k)=e_{k}$.

Note that the tree struct $i_{i}^{S}$ captures the order of the substitutions which are necessary to obtain $U_{i}$. To show that every equational graph is suffix-representable, we observe that an equational graph, say the graph of $\operatorname{val}\left(U_{i}\right)$, can be seen as the limit of a succession of graphs. In fact, let the n-graphs $G_{j}$ be defined as:

- $G_{1}=\operatorname{val}\left(e_{i}\right)$;

- $G_{j+1}=G_{j}\left[u_{1} \leftarrow \operatorname{val}\left(e_{1}\right), \ldots, u_{m} \leftarrow \operatorname{val}\left(e_{m}\right)\right]$ for every $j>0$.

Let $\bar{G}_{j}$ be the graph obtained from the graph of $G_{j}$, by deleting the edges labelled by the unknowns. Trivially, for all $j>0$ the relation $\bar{G}_{j} \subseteq \bar{G}_{j+1}$ holds and the graph of $\operatorname{val}\left(U_{i}\right)$ is equal to $\lim _{j} \bar{G}_{j}$.

Let $l a b^{\prime}$ be defined in such a way that if there exists an $h$ such that $v_{1}, \ldots, v_{k} \in$ $V_{h}$, then $l a b^{\prime}\left(x v_{1}, \ldots, x v_{k}\right)$ is equal to $l a b_{h}\left(v_{1}, \ldots, v_{k}\right)-\left\{u_{1}, \ldots, u_{m}\right\}$, otherwise $l a b^{\prime}\left(x v_{1}, \ldots, x v_{k}\right)$ is $\mathbf{0}$. Moreover, let $L^{\prime}=\bigcup_{x \in \operatorname{dom}\left(\operatorname{struct}_{i}^{S}\right)} x V_{x}$ where $V_{x}$ is $V_{h}$ if $\operatorname{struct}_{i}^{S}(x)=e_{h}, L_{n}^{\prime}$ be the set $\left\{x \in L^{\prime} /|x|=n\right\}$ and $E$ be the set of edges $\left\{\left(v_{1}, \ldots, v_{k}\right) \in \bigcup_{j=1}^{m} E_{j} / l a b^{\prime}\left(v_{1}, \ldots, v_{k}\right) \neq \mathbf{0}\right\}$. As a consequence of a substitution, some vertices are collapsed according to an equivalence relation. Thus the substitutions given by struct $t_{i}^{S}$ induce an equivalence relation on $L^{\prime}$. We define a mapping sho $: L^{\prime} \rightarrow L^{\prime}$ with the meaning that sho $(x)$ is the shortest string in the equivalence class of $x$ and this string will denote the vertex obtained by gluing all the vertices in this equivalence class. Then, by the substitutions a vertex $x$ will be replaced by the vertex $\operatorname{sho}(x)$. Formally, for all $x \in \operatorname{dom}\left(\operatorname{struct}_{i}^{S}\right)$, $h \in \operatorname{unk}\left(\operatorname{struct}_{i}^{S}(x)\right)$ and $v \in V_{h}$ :

- $\operatorname{sho}(x h v)=\operatorname{sho}\left(x \pi_{k}^{h}\left(e_{j}\right)\right)$, if $\operatorname{struct}_{i}^{S}(x)=e_{j}$ and $v=\operatorname{src}_{h}(k)$, and 
- $\operatorname{sho}(x v)=x v$, otherwise.

In the following we will denote with $\operatorname{sho}_{n}^{-1}(x)$ the set $\operatorname{sho} o^{-1}(x) \cap L_{n}^{\prime}$ and with $L_{n}$ and $P_{n}$ the sets:

- $L_{1}=V_{i}$ and $P_{1}=E_{i}$;

- $L_{n+1}=L_{n} \cup \operatorname{sho}\left(L_{n+1}^{\prime}\right)$ and $P_{n+1}=P_{n} \cup$ $\left\{\operatorname{suf}\left(\operatorname{sho}\left(x_{1}\right), \ldots, \operatorname{sho}\left(x_{k}\right)\right) / x_{1}, \ldots, x_{k} \in L_{n+1}^{\prime}\right.$ and $\left.\operatorname{suf}\left(x_{1}, \ldots, x_{k}\right) \in E\right\}$.

Furthermore we denote with $l a b_{n}^{\prime}$ the total mapping defined as:

$l a b_{1}^{\prime}=l a b_{i}-\left\{u_{1}, \ldots, u_{m}\right\}$ and

$l a b_{n+1}^{\prime}\left(\operatorname{suf}\left(x_{1}, \ldots, x_{k}\right)\right)=\operatorname{lab} b_{n}^{\prime}\left(\operatorname{suf}\left(x_{1}, \ldots, x_{k}\right)\right)+\sum_{y_{j} \in \operatorname{sho}_{n+1}^{-1}\left(x_{j}\right)} l a b^{\prime}\left(y_{1}, \ldots, y_{k}\right)$

for all $x_{1}, \ldots, x_{k} \in L_{n+1}$. Note that, directly from the above definitions, it holds that $L_{n} \subseteq L_{n+1}, P_{n} \subseteq P_{n+1}$ and $l a b_{n}^{\prime}\left(x_{1}, \ldots, x_{k}\right) \leq l a b_{n+1}^{\prime}\left(x_{1}, \ldots, x_{k}\right)$. The above definitions are used in the proof of the following theorem:

Theorem 3.1. Every equational graph is suffix-representable.

Proof. Let us consider the graph of $\operatorname{val}\left(U_{i}\right)$ and let $L$ be the language $\operatorname{sho}\left(L^{\prime}\right)$, $P$ be the set $\left\{\operatorname{suf}\left(\operatorname{sho}\left(x v_{1}\right), \ldots, \operatorname{sho}\left(x v_{k}\right)\right) / x \in \operatorname{dom}\left(\operatorname{struct}_{i}^{S}\right)\right.$ and $\left(v_{1}, \ldots, v_{k}\right) \in$ $E\}$ and $l a b$ be defined as $\operatorname{lab}\left(\operatorname{suf}\left(x_{1}, \ldots, x_{k}\right)\right)=\sum_{y_{j} \in s^{-1}\left(x_{j}\right)} l a b^{\prime}\left(y_{1}, \ldots, y_{k}\right)$. By Proposition 2.8 the graph $\operatorname{gra}(L$, suffix, $P, l a b)$ is the limit of the succession $\left\{\operatorname{gra}\left(L_{n} \text {, suffix, } P_{n}, l a b_{n}^{\prime}\right)\right\}_{n>0}$. Moreover, it is possible to prove that the graph $\bar{G}_{n}$ is isomorphic to $\operatorname{gra}\left(L_{n}, \operatorname{suffix}, P_{n}, l a b_{n}^{\prime}\right)$. As a consequence of the above fact and by Proposition 2.2, we obtain that the graph $\operatorname{gra}(L$, suffix, $P, l a b)$ is isomorphic to the graph of $\operatorname{val}\left(U_{i}\right)$. Further, note that the language $L$ is regular and prefix-free. In fact, by the definition of sho, $L$ can be seen as $V_{i} \cup \bigcup_{x \in \operatorname{dom}_{\text {(struct }}^{S} \text { ) }} x V_{x}$ where $V_{x}$ is the set $\left\{v \in V_{h} / v \neq \operatorname{src}_{h}(i) \forall i=1, \ldots, n_{h}\right.$ and $\left.e_{h}=\operatorname{struct}_{i}^{S}(x)\right\}$.

To complete the proof we have only to show that $P$ is the finite union of the languages $P^{\prime}$ such that $P^{\prime}$ is regular in parallel and $\left|\operatorname{lab}\left(P^{\prime}\right)\right|=1$. At this aim, we define the mapping $\phi$ as $\phi\left(v_{1}, \ldots, v_{k}\right)=\bigcup_{j=1}^{m}\left\{\left(w_{1}, \ldots, w_{k}\right) \in\left(V_{j}\right)^{k} / \exists h\right.$ such that $w_{h} \neq \operatorname{src}_{j}(l)$ for all $l=1, \ldots, n_{j}$ and $\exists x, y \in \operatorname{dom}\left(\operatorname{struct}_{i}^{S}\right)$ such that $\operatorname{sho}\left(x v_{l}\right)=$ $\left.\operatorname{sho}\left(y w_{l}\right), \forall l=1, \ldots, k\right\} . \phi\left(v_{1}, \ldots, v_{k}\right)$ is the set of the tuples $\left(w_{1}, \ldots, w_{k}\right) \in$ $\left(V_{j}\right)^{k}$, for $j=1, \ldots, m$, such that for some $x$ and $y$ the words $x v_{l}$ and $y w_{l}$ are replaced by the same word in $L$ and at least one among $y w_{1}, \ldots, y w_{k}$ belongs to $L$. This implies that for all $\left(w_{1}, \ldots, w_{k}\right) \in \phi(E)$ and for all the tuples $\left(x_{1}, \ldots, x_{k}\right)$, $\left(x_{1}^{\prime}, \ldots, x_{k}^{\prime}\right)$ belonging to $L^{k}$ such that there exist $x, x^{\prime}$ with $\operatorname{sho}\left(x w_{l}\right)=x_{l}$ and $\operatorname{sho}\left(x^{\prime} w_{l}\right)=x_{l}^{\prime}$, it holds that $\operatorname{lab}\left(\operatorname{suf}\left(x_{1}, \ldots, x_{k}\right)\right)=\operatorname{lab}\left(\operatorname{suf}\left(x_{1}^{\prime}, \ldots, x_{k}^{\prime}\right)\right)$. Then, we have that $P=E_{i} \cup\left(\bigcup_{\left(v_{1}, \ldots, v_{k}\right) \in \phi(E)} P\left(v_{1}, \ldots, v_{k}\right)\right)$, where $P\left(v_{1}, \ldots, v_{k}\right)=$ $\left\{\operatorname{suf}\left(\operatorname{sho}\left(x v_{1}\right), \ldots, \operatorname{sho}\left(x v_{k}\right)\right) / x \in \operatorname{dom}\left(\operatorname{struct} t_{i}^{S}\right)\right\}$, and $\left|\operatorname{lab}\left(P\left(v_{1}, \ldots, v_{k}\right)\right)\right|=1$ holds for all $\left(v_{1}, \ldots, v_{k}\right) \in \phi(E)$. The basic idea in the construction of the finite automaton $A\left(v_{1}, \ldots, v_{k}\right)$ accepting $P\left(v_{1}, \ldots, v_{k}\right)$, for every $\left(v_{1}, \ldots, v_{k}\right) \in \phi(E)$, is: $A\left(v_{1}, \ldots, v_{k}\right)$ saves in its states, step by step and in the reverse mode, the sequence of the replacements (see the recursive definition of $s h o$ ) that leads from a given $\operatorname{suf}\left(x_{1}, \ldots, x_{k}\right)$ to $\left(x v_{1}, \ldots, x v_{k}\right)$ for some $x$ (note that if $x_{j}$ is the longest word among $x_{1}, \ldots, x_{k}$ then $\left.x v_{j}=x_{j}\right)$; then, $A\left(v_{1}, \ldots, v_{k}\right)$ accepts a word if and only if it is able to rebuilt that sequence (i.e. such a sequence exists) and, thus, if and only if that word belongs to $P\left(v_{1}, \ldots, v_{k}\right)$. 
Notice that the above theorem holds even if we restrict $P$ to the finite union of sets given by the product of regular languages. Moreover, the given proof provides a way to obtain a description of every equational graph in the notation of suffix-representable graphs. For example, if we consider the system $u=\hat{\sigma}_{f}\left(\hat{\theta}_{\delta}(b \hat{\oplus} a \hat{\oplus} c \hat{\oplus} u)\right)$ where $\delta$ is $\{(1,3),(2,6),(4,7),(5,8)\}$ and $f:\{1,2\} \rightarrow$ $\{1,2,3,4,5,6,7,8\}$ is defined as $f(i)=i$ for $i=1,2$, then the graph obtained in that way is the one in Figure 1.

The vice versa of the above theorem is not true. In fact there are graphs which are suffix-representable but not equational.

Lemma 3.2. Let $G=(V, E, l a b, s r c)$ be an $n$-graph and $G^{\prime}=\left(V^{\prime}, E^{\prime}, l a b^{\prime}, s r c^{\prime}\right)$ be a subgraph of $G$ such that:

a) $V^{\prime}$ is infinite and

b) for every $v, v^{\prime} \in V^{\prime}$ there exists $\left(v_{1}, \ldots, v_{k}\right) \in E^{\prime}$ such that $\left(v_{1}, \ldots, v_{k}\right)$ is incident on $v$ and $v^{\prime}$.

It holds that the graph of $G$ is not equational.

Proof. Let $(\Delta, \tau)$ be a ranked alphabet, $G_{i}=\left(V_{i}, E_{i}, l a b_{i}, \operatorname{src}_{i}\right)$, for $i=1,2$, be $n_{i}$-graphs, $\left(v_{1}, \ldots, v_{k}\right) \in E_{1}$, and $a \in \Delta$ be such that $l a b_{1}\left(v_{1}, \ldots, v_{k}\right)(a)>0$ and $\tau(a)$ is the type of $G_{2}$. For every $v \in V_{2}$ which is not a source of $G_{2}$ and $v^{\prime} \in V_{1}$ such that there exists an edge of $G_{1}\left[\left(\left(v_{1}, \ldots, v_{k}\right), a\right) \leftarrow G_{2}\right]$ which is incident on both $v$ and $v^{\prime}$, it trivially holds that $v^{\prime}$ is a vertex of the edge $\left(\left(v_{1}, \ldots, v_{k}\right), a\right)$.

Now, let $S=\left\langle u_{1}=e_{1}, \ldots, u_{m}=e_{m}\right\rangle$ be a system of graph equations fulfilling the Greibach condition and $\left(U_{1}, \ldots, U_{m}\right)$ be the solution of $S$. By the above observation, for every $i=1, \ldots, m$ and for every subgraph $G^{\prime}=\left(V^{\prime}, E^{\prime}, l a b^{\prime} s r c^{\prime}\right)$ of $\operatorname{val}\left(U_{i}\right)$ fulfilling the property b), it holds that $\left|V^{\prime}\right| \leq \max \left\{\left|V_{h}\right| / V_{h}\right.$ is the set of vertices of $\operatorname{val}\left(e_{h}\right)$ for $\left.h=1, \ldots, m\right\}$.

Corollary 3.3. The class of the equational graphs is strictly contained in the class of the suffix-representable graphs.

Proof. The result is a direct consequence of Theorem 3.1, Lemma 3.2 and the fact that all the representable graphs containing $\operatorname{gra}(L$, suffix, $P, l a b)$ as a subgraph, where $L=a^{*} b, P=\{(b, a)\}$ and $l a b(a, b) \neq \mathbf{0}$, satisfy the conditions of Lemma 3.2.

\section{Simple GRAPHS vs. SUFFIX-REPRESENTABlE GRAPHS}

In [5] Caucal defines a family of simple graphs whose monadic theory is linearly reducible to the monadic second-order theory of two successors. This class of graphs strictly contains the equational simple graphs of Courcelle [7], which is a proper subclass of the class considered in Section 3, and coincides with the class of equational graphs defined by Barthelmann [2]. In this section we briefly recall the definition of the Caucal graphs and we compare this class with the class of suffix-representable by graphs. To this aim we restrict to simple graphs. 
Let $\Delta$ be an alphabet, with $\bar{\Delta}$ we denote the alphabet $\{\bar{a} / a \in \Delta\}$. Let $\Delta, \Delta^{\prime}$ be two alphabets, we define a substitution as a mapping $h: \Delta^{*} \longrightarrow 2^{\Delta^{\prime *}}$ such that: $h(\varepsilon)=\{\varepsilon\}$ and $h(u v)=h(u) h(v)$ for every $u, v \in \Delta^{*}$. Let $G=(V, l a b)$ be a $\Delta$ labelled graph and $h: \Delta^{*} \longrightarrow 2^{(\Delta \cup \bar{\Delta})^{*}}$ be a substitution, we denote with $h^{-1}(G)$ the graph $\left(V, l a b^{\prime}\right)$ where $l a b^{\prime}: V^{2} \rightarrow M S(\Delta)$ is a total mapping such that, for every $a \in \Delta$ and $u, v \in V, l a b^{\prime}(u, v)(a)=1$ if and only if there exist $v_{1}, \ldots, v_{k} \in V$ and $b_{1} \ldots b_{k-1} \in h(a)$ such that $u=v_{1}, v=v_{k}$ and for $i=1, \ldots, k-1$ :

- $\operatorname{lab}\left(v_{i}, v_{i+1}\right)\left(b_{i}\right)=1$ if $b_{i} \in \Delta$ and

- $l a b\left(v_{i+1}, v_{i}\right)(b)=1$ if $b_{i}=\bar{b}$.

Let $\Lambda_{\Delta}$ be the graph $\left(\Delta^{*}, l a b\right)$ where $\operatorname{lab}(u, v)(a)=1$ if and only if $v=u a$ for every $u, v \in \Delta^{*}$ and $a \in \Delta$, that is $\Lambda_{\Delta}$ is the complete infinite $|\Delta|$-ary tree. Let $\mathcal{L}$ be a class of languages, the class of graphs $R E C_{\mathcal{L}}$ is defined as the set of graphs $h^{-1}\left(\Lambda_{\Delta}\right)_{\mid L}$ such that $h: \Delta^{*} \rightarrow \mathcal{L} \cap 2^{(\Delta \cup \bar{\Delta})^{*}}$ is a substitution and $L$ is a regular language on $\Delta$. In [5] the classes $R E C_{F i n}$ and $R E C_{R a t}$ are considered, where for Fin and Rat denote respectively the classes of the finite and regular languages.

In the following two examples we exhibit two infinite graphs which are suffixrepresentable but are not in $R E C_{\text {Rat }}$.

Example 4.1. Let $G=(N \times N, l a b)$ be such that:

- $E_{G}=\{((i, j),(i+1, j)) / i, j \in N\} \cup\{((i, j),(i, j+1)) / i, j \in N\}$;

- $\operatorname{lab}((i, j),(i+1, j))=\{b\}$ and $\operatorname{lab}((i, j),(i, j+1))=\{a\}$.

Then, $G$ is suffix-representable. In fact, let $G^{\prime}$ be the graph $\operatorname{gra}\left(L\right.$, suffix, $\left.P, l a b^{\prime}\right)$ where $L=z^{*} y^{*} x, P=\left\{\left(y^{i} x, z y^{i} x\right) / i \geq 0\right\} \cup\{(x, y x)\}$ and $l a b^{\prime}\left(y^{i} x, z y^{i} x\right)=\{b\}$ and $l a b^{\prime}(x, y x)=\{a\}$ (see Fig. 2). It is easy to prove that $G^{\prime}$ is isomorphic to $G$.

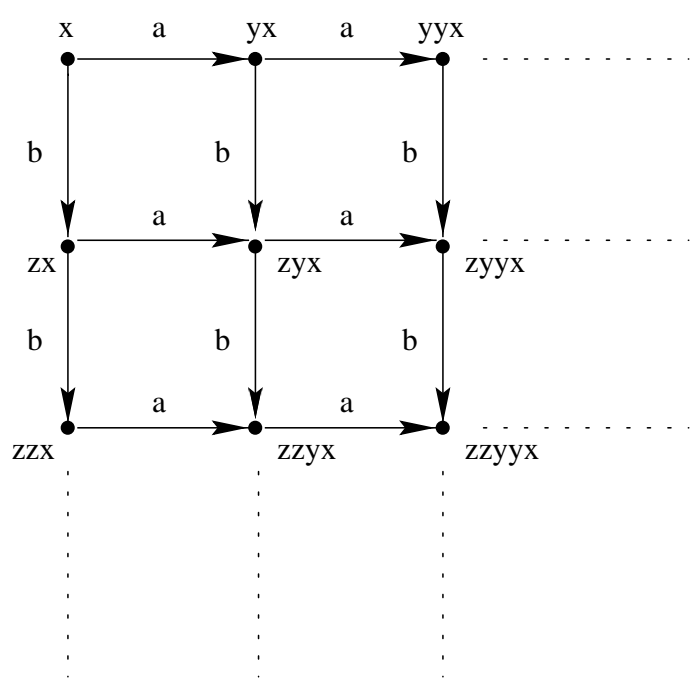

Figure 2. A graphical representation of $G^{\prime}$. 
Example 4.2. Let $G=(N, l a b)$ be such that:

- $E_{G}=\{(i, i+1) / i \in N\} \cup\left\{\left(2^{i+1}-1,2^{i+1}-1\right) / i \in N\right\}$;

- $l a b(i, j)=\{a\} \forall(i, j) \in E$.

Then, $G$ is suffix-representable. In fact, let $G^{\prime}$ be the graph $\operatorname{gra}\left(L\right.$, suffix, $\left.P, l a b^{\prime}\right)$ where $L=x(y+z)^{*} x+x y^{+} v, P=\{(y v, z x),(y x, z x),(v, v)\} \cup\left\{\left(x z^{i} x, x y^{i+1} v\right)\right) / i \geq$ $0\} \cup\left\{\left(y z^{i} x, z y^{i} x\right) / i \geq 0\right\}$ and $l a b^{\prime}(u, w)=\{a\} \forall(u, w) \in P$ (see Fig. 3). It is easy to prove that $G^{\prime}$ is isomorphic to $G$.

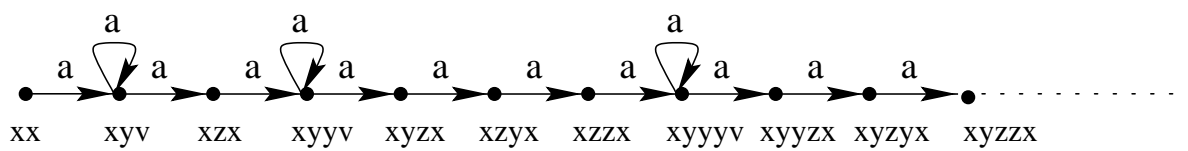

FiguRE 3. A graphical representation of $G^{\prime}$.

Theorem 4.3. $R E C_{R a t}$ coincides with the class of suffix-representable simple graphs gra $(L$, suffix, $P, l a b)$ such that $P$ is the finite union of sets of type $L_{1} \times L_{2}$ for regular languages $L_{1}$ and $L_{2}$, and is strictly contained in the class of suffixrepresentable simple graphs.

Proof. The first assertion directly follows from the definitions. For the second assertion, we observe that by the characterization of $R E C_{R a t}$ shown in [5], we have that $R E C_{R a t}$ is contained in the class of suffix-representable graphs. The strict inclusion is a consequence of the fact that the graphs in the Examples 4.1 and 4.2 are suffix-representable and are not in $R E C_{R a t}$ [5].

Theorem 4.4. $R E C_{F i n}$, the class of equational simple graphs with finite degree, coincides with the class of simple graphs gra $(L$, suffix, $P, l a b)$, where $L$ is a regular prefix-free language and $P$ is finite.

Proof. Directly from the definitions.

\section{The CLASS OF INFIX-REPRESENTABLE GRAPHS}

In this section we study the class of infix-representable graphs. We start giving an interesting property which relates a graph operation in this class to a language operation. Then we compare the infix-representable graphs to the suffixrepresentable graphs. Finally, we conclude solving some typical decision problems for graphs in the class of infix-representable graphs.

Graph substitution corresponds to a language concatenation for graphs represented via regular languages and the infix function. Let $G=(V, l a b)$ and $G_{v}=\left(V_{v}, l a b_{v}\right)$, for $v \in V$, be graphs. Then, the graph obtained by substituting $G_{v}$ for $v$ in $G$, denoted by $G\left[v \leftarrow G_{v}\right]_{v \in V}$, is the graph $\left(V^{\prime}, l a b^{\prime}\right)$ where:

- $V^{\prime}=\bigcup_{v \in V}\{v\} V_{v}$; 
- for $x_{1}, \ldots, x_{k} \in V$ and $y_{i} \in V_{x_{i}}, i=1, \ldots, k, l a b^{\prime}\left(x_{1} y_{1}, \ldots, x_{k} y_{k}\right)$ is equal to $l a b_{x_{1}}\left(y_{1}, \ldots, y_{k}\right)$, if $x_{1}=\ldots=x_{k}$, and is equal to $\operatorname{lab}\left(x_{1}, \ldots, x_{k}\right)$, otherwise.

This graph substitution is said uniform if we substitute the same unique graph for every vertex $v$. The following theorem states that uniform graph substitution corresponds to marked language concatenation in the representation of the graph.

Proposition 5.1. Let $G=\operatorname{gra}(L$, infix, $P$, lab $), G_{x}=\operatorname{gra}\left(L_{x}\right.$, infix, $P$, lab $)$ for $x \in L$ and $\$$ be a symbol not in the alphabet of $L$ and $L_{x}$ for any $x \in L$. It holds that $G\left[x \leftarrow G_{x}\right]_{x \in L}$ is isomorphic to gra $\left(L^{\prime}\right.$, infix, $P$, lab $)$, where $L^{\prime}$ is the language $\bigcup_{x \in L}\{x \$\} L_{x}$. Moreover, if $L^{\prime \prime}=L_{x}$ for every $x \in L$ then $L^{\prime}=L\{\$\} L^{\prime \prime}$.

Proof. We observe that the graph $G\left[x \leftarrow G_{x}\right]_{x \in L}$ consists of disjoint subgraphs each of them is induced by the vertices of some $G_{x}$. Moreover, the edges connecting two vertices belonging to two distinct such subgraphs correspond to the $G$ edges connecting the vertices that have been replaced by these subgraphs. We also observe that there is an obvious bijection from $x \$ L_{x}$ to $L_{x}$ and that $\operatorname{gra}\left(x \$ L_{x}\right.$, infix, $\left.P, l a b\right)$ is isomorphic to $\operatorname{gra}\left(L_{x}\right.$, infix, $\left.P, l a b\right)$. Furthermore, in graph $\operatorname{gra}\left(L^{\prime}\right.$, infix, $\left.P, l a b\right)$ there is an edge connecting a vertex of $\operatorname{gra}\left(x \$ L_{x}\right.$, infix, $P$, lab $)$ to a vertex of $\operatorname{gra}\left(y \$ L_{y}\right.$, infix, $\left.P, l a b\right)$ for $x, y \in L$ if and only if there is an edge in $G$ connecting $x$ to $y$. Thus we get that $G\left[x \leftarrow G_{x}\right]_{x \in L}$ is isomorphic to $\operatorname{gra}\left(L^{\prime}\right.$, infix, $\left.P, l a b\right)$.

Notice that even if we apply many substitutions we do not add more than one new symbol.

\subsection{SufFIX-REPRESENTABLE GRAPHS vs. INFIX-REPRESENTABLE GRAPHS}

In this section we compare the class of the suffix-representable graphs to that of the infix-representable graphs. We first observe that the following property holds for the suffix-representable graphs.

Proposition 5.2. Let $G=\left(V, l a b^{\prime}\right)$ be the suffix-representable graph denoted as $\operatorname{gra}(L$, suffix, $P$, lab $)$. It holds that $\left|l a b^{\prime}\left(E_{G}\right)\right| \leq|\operatorname{lab}(P)|$.

Proof. By the definition of the function suffix, we have that each tuple has exactly a suffix. Thus by definition of suffix-representable graph we get that the number of different labels of a suffix-representable graph cannot be greater than the number of different labels for $P$.

Notice that the strict inequality holds in the above statement if some of the tuples in $P$ are not suffixes of any of the tuples of $G$ vertices. Moreover, this property may not hold for the infix-representable graphs as shown by the following example:

Example 5.3. Let $G=\operatorname{gra}\left(a^{*} c+b^{*} c\right.$, infix, $\left.P=\left\{\left(a^{n}, b^{n}\right) / n \in N\right\}, l a b\right)$ where $|\operatorname{lab}(P)|=1$ be a infix-representable graph. The graph $G$ is the graph $\left(a^{*} c+\right.$ $\left.b^{*} c, l a b^{\prime}\right)$ where $E_{G}=\left\{\left(a^{n} c, b^{m} c\right) / n, m \in N\right\}, l a b^{\prime}\left(a^{n} c, b^{m} c\right)=\sum_{i=1}^{\min (n, m)} l a b\left(a^{i}, b^{i}\right)$ and, then, $\left|l a b^{\prime}\left(E_{G}\right)\right|=\infty$. 
From the above example and lemma, it is clear that the suffix-based representation is not enough to express all the infix-representable graphs. Thus we have the following theorem:

Theorem 5.4. The class of suffix-representable graphs is strictly contained into the class of infix-representable graphs.

Proof. Let $\Sigma$ be an alphabet, $L \subseteq \Sigma^{*}$ and $P \subseteq \bigcup_{i=1}^{r}\left(\Sigma^{*}\right)^{i}$. Consider $G=$ $\operatorname{gra}(L$, suffix, $P, l a b)$ and $\$ \notin \Sigma$, then we have $G=\operatorname{gra}\left(L^{\prime}\right.$, infix, $\left.P^{\prime}, l a b^{\prime}\right)$ where $L^{\prime}=L\{\$\}, P^{\prime}=\left\{\left(x_{1} \$, \ldots, x_{k} \$\right) /\left(x_{1}, \ldots, x_{k}\right) \in P\right\}$ and $l a b^{\prime}\left(x_{1} \$, \ldots, x_{k} \$\right)=$ $l a b\left(x_{1}, \ldots, x_{k}\right)$. The strict inclusion is a consequence of Proposition 5.2 and the Example 5.3.

It is worth noticing that, depending on the graph, specifying the infix-based representation could be shorter than the suffix-based one or vice versa. Anyway, from the above proof the minimal infix-based representation is, in the worst case, slightly more expensive than the minimal suffix-based one (there is just a new symbol to add). On the other side, the suffix based representation could be much more expensive than the infix-based one and the following example gives some hint on this:

Example 5.5. Let $n$ be a positive integer and $T_{n}$ be a complete $n$-ary 1-level tree whose edges are labelled by $a$. We define the graph $G_{n}$ as the graph consisting of infinite distinct components all isomorphic to $T_{n}$. Let $L_{n}=x^{*} y+x^{*}\left\{z^{i} w / 1 \leq\right.$ $i \leq n\}$. Trivially, $G_{n}=\operatorname{gra}\left(L_{n}\right.$, infix, $\left.\{(y, z)\}, l a b\right)$ where $\operatorname{lab}(y, z)=\{a\}$. We observe that any suffix-based representation for $G_{n}$ cannot have less than $n$ pairs in $P$ (less than $n$ leads to insert edges among the components isomorphic to $T_{n}$ ). Then, a reasonable suffix-based representation for $G_{n}$ is $\left(L_{n}\right.$, suffix, $\left\{\left(y, z^{i} w\right) / 1 \leq\right.$ $\left.i \leq n\}, l a b^{\prime}\right)$ where $l a b^{\prime}\left(y, z^{i} w\right)=\{a\}$.

The interesting fact, stated by the following theorem, is that the weakness of the suffix-based representation with respect to the infix-based one is only related to the labelling. These two classes coincide on unlabelled graphs. Clearly, an $f$-representable unlabelled graph $\operatorname{gra}(L, f, P)$ is the unlabelled graph such that $L$ is the set of vertices and $\left\{\left(x_{1}, \ldots, x_{k}\right) \in V^{k} / k \geq 1, f\left(x_{1}, \ldots, x_{k}\right) \cap P \neq \emptyset\right\}$ is the set of hyper-edges.

Proposition 5.6. The class of suffix-representable unlabelled graphs is equivalent to the class of infix-representable unlabelled graphs.

The class of suffix-representable simple graphs is equivalent to the class of infixrepresentable simple graphs.

Proof. Consider the first assertion. One inclusion directly comes from Theorem 5.4. For the other inclusion consider $G=\operatorname{gra}\left(L\right.$, infix, $\left.\bigcup_{i=1}^{m} P_{i}\right)$ such that the property stated in Proposition 2.9 holds. We get that $G=\operatorname{gra}\left(L\right.$, suffix, $\left.P^{\prime}\right)$ where $P^{\prime}$ is the union of the $P_{i}$ 's such that $\left(x_{1}, \ldots, x_{k}\right) \in P_{i}$ implies $x_{1}=\ldots=x_{k}$, and of the $P_{i}^{\prime}=\left\{\left(x_{1} y_{1}, \ldots, x_{k} y_{k}\right) / y_{1}, \ldots, y_{k} \in \Sigma^{*},\left(x_{1}, \ldots, x_{k}\right) \in P_{i}\right\}$ where $P_{i}$ is such that for $\left(x_{1}, \ldots, x_{k}\right) \in P_{i}$ there is not a $y \in \Sigma^{+}$such that $x_{i}=y x_{i}^{\prime}$ for $i=1, \ldots, k$. In a similar way we can prove the second assertion. 
Restricting to a finite language $P$, the infix-based notation is strictly more expressive than the suffix-based one on both labelled and unlabelled graphs, as shown in the following theorem:

Theorem 5.7. The class of graphs gra $(L$, suffix, $P, l a b)$, where $L$ is a regular prefix-free language and $P$ is finite, is strictly contained in the class of graphs $\operatorname{gra}\left(L^{\prime}\right.$, infix, $P^{\prime}$, la $\left.b^{\prime}\right)$, where $L^{\prime}$ is a regular prefix-free language and $P^{\prime}$ is finite.

Proof. By the same construction we used in the proof of Theorem 5.4, it holds that all the $\operatorname{gra}(L$, suffix, $P, l a b)$ with a finite $P$ belong to the class of graphs $\operatorname{gra}\left(L^{\prime}\right.$, infix, $\left.P^{\prime}, l a b^{\prime}\right)$ with a finite $P^{\prime}$. The strict inclusion is consequence of the fact that $\operatorname{gra}(L$, infix, $P, l a b)$ has a finite degree if $P$ is finite and, denoted as $G_{n}$ the graphs defined in Example 5.5, we have that $\lim _{n} G_{n}=\operatorname{gra}\left(x^{*} z^{*} y\right.$, infix, $\left.\{y, z\}, l a b\right)$.

\subsection{CheCKING PROPERTIES FOR INFIX-REPRESENTABLE GRAPHS}

In this section we deal with some typical problems on graphs and, for the sake of simplicity, we briefly discuss some algorithms to solve them on simple graphs which are infix-representable, without self-loops and whose edges have all length 2. Since in this section we are not interested in the labelling of the edges, we will simply suppose that all the edges have the same labelling, that is the set $P$ is such that $|l a b(P)|=1$. Furthermore, we will refer to well known results in the field of finite automata without recalling them (see [12] for a reference). In the following, we will denote with $G$ an infix-representable graph $\operatorname{gra}(L$, infix, $P, l a b)$ and with $|A|$ and $|v|$ we denote the size of a given automaton $A$ and the length of a given word $v$, respectively.

Let $A_{L}$ and $A_{P}$ be respectively the finite automata that recognize $L$ and $P$ (note that $A_{L}$ is a usual finite automaton while $A_{P}$ is a 2-FA). From these two automata we can obtain a 2 -FA $A_{E}$ recognizing the set of the edges of $G$, that is the set of pairs $(u, v)$ such that $u, v \in L$ and $\inf (u, v) \cap P \neq \emptyset$. Informally, $A_{E}$, scanning the input pair, checks that the common prefix of the pair is a legal prefix for words in $L$, then on the first difference it simulates $A_{P}$ and continues the simulation of $A_{L}$ on both the sides of the input still to be scanned. Note that, the corresponding construction brings to a nondeterministic 2-FA that we denote with $A_{E}$ and whose size $\left|A_{E}\right|$ is quadratic in the size of $A_{L}$ and linear in the size of $A_{P}$, that is $\left|A_{E}\right|=O\left(\left|A_{L}\right|^{2}\left|A_{P}\right|\right)$. The nondeterminism of $A_{E}$ comes out from the fact that $P$ accepts also pairs of words which have different lengths. Then, the following theorem holds:

Theorem 5.8. If a graph $G=(V, l a b)$ is infix-representable then there exists a 2-FA $A_{E}$ such that $L\left(A_{E}\right)=E_{G}$.

The first problem we consider is the adjacency problem, so stated: "given two words $u, v$ do they represent two adjacent vertices in $G$ ?"

To answer this question it is enough to check for the membership of $(u, v)$ to the language accepted by $A_{E}$ and this can be done in linear time in the size of $A_{E}$ and in the size of $(u, v)$, that is $O\left(\left|A_{E}\right| \max (|u|,|v|)\right)$. 
Another interesting aspect of the infix-representable graphs is that the set of vertices which have entering edges and the set of the vertices which have exiting edges are both regular. In fact, it is possible to construct in time $O\left(\left|A_{E}\right|\right)$ the automata recognizing these languages. We omit the details of these constructions since they are simply the automata accepting the projections on the first and on the second components of $L\left(A_{E}\right)$. In the following, we denote with $A_{\text {in }}$ the automaton accepting the vertices with entering edges and with $A_{\text {out }}$ the other one. We can use these automata to answer questions such as the existence of a source, a sink, a transient (i.e., with in-going and out-going edges), or an isolated vertex in the graph. In fact, we can check the existence of a source just checking the nonemptyness of $\overline{L\left(A_{\text {in }}\right)} \cap L\left(A_{\text {out }}\right)$ and the other three problems can be handled in an analogous way. Except for the transient vertices, the corresponding algorithms are exponential since we need to construct the automata accepting the complementation of a language recognized by a nondeterministic finite automaton. In the case of transient vertices the algorithm takes time $O\left(\left|A_{\text {in }}\right|\left|A_{\text {out }}\right|\right)$. If we are just interested in checking these properties for a given vertex $v$, we only have to check if $v$ is a member of $L\left(A_{\text {in }}\right)$ and $L\left(A_{\text {out }}\right)$ and, in the worse case, this takes time $O\left(\left|A_{E}\right||v|\right)$.

Analogous algorithms can be used to determine the degree of a given vertex $v$ in a graph $G$. (Recall that the degree of a vertex $v$ is the number of the edges incident on $v$ and the degree of a graph is the maximum degree of its vertices.) In fact, we can construct the automaton accepting all the pairs $(v, u),(u, v) \in L\left(A_{E}\right)$ starting from $A_{E}$ (it takes time $O\left(|v|\left|A_{E}\right|\right)$ ), and, then, check for the finiteness of the accepted language. If this language is finite we can count the words in it and, since the corresponding automaton is nondeterministic, this takes exponential time.

Now we consider properties concerning the degree of a graph. In the following we denote with $n$ the number of states of $A_{E}$ and with $\operatorname{deg}(v)$ the degree of a given node $v$. As a direct consequence of results on regular languages we have the following remark:

Remark 5.9. $\quad$ 1. The degree of $G$ is 0 iff $L\left(A_{E}\right)$ is empty.

2. The edges of the graph $G$ are incident only to nodes $v$ such that $|v|<n$ iff $L\left(A_{E}\right)$ is finite.

The following theorem states a necessary and sufficient condition for the infiniteness of the degree of an infix-representable graph. From this result an algorithm, running in $O\left(2^{\left|A_{E}\right|}\right)$ time, can be obtained to decide whether the degree of a graph is infinite or not.

Theorem 5.10. $G$ has infinite degree iff there exists $v \in L$ such that $|v|<n$ and the degree of $v$ is infinite.

Proof. Given a 2-FA $A$, we denote an accepting run of $A$ as a triple $\left(s, w_{1}, w_{2}\right)$ where $s$ is a sequence of states (starting with the initial state and ending with a final state) which are entered by $A$ when reading the pair of words $\left(w_{1}, w_{2}\right)$. With 
$w o_{1}$ and $w o_{2}$ we denote the projection of the second and the third component of the run, respectively.

Suppose that $G$ has an infinite degree. Let $u \in L$ such that $\operatorname{deg}(u)=\infty$ and $|u|$ is minimal. Suppose that $|u| \geq n$. As a consequence, there exists a set $R$ of $A_{E}$ accepting runs such that:

- $|R|=\infty$;

- $\forall r \in R$, either $\left(w o_{1}(r)=u\right.$ and $\left.\left|w o_{2}(r)\right|>|u|\right)$ or $\left(w o_{2}(r)=u\right.$ and $\left|w o_{1}(r)\right|>$ $|u|)$;

- $\forall r, r^{\prime} \in R$, the first component of $r(i)$ is equal to the first component of $r^{\prime}(i)$ for $i=0, \ldots,|u|$.

Since $|u| \geq n$, an $A_{E}$ cycle occurs in the first $|u|$ triples of any $r \in R$. Thus by cutting such cycles in the runs belonging to $R$, it is possible to obtain a set of runs $R^{\prime}$ that satisfy the above properties with respect to a word $w \in L$ such that such that $\operatorname{deg}(w)=\infty$ and $|w|<|u|$. Hence for all $|u| \geq n, u \in L$, such that $\operatorname{deg}(u)=\infty$ there exists a $w \in L,|w|<|u|$, such that $\operatorname{deg}(w)=\infty$ and this contradicts the minimality of $|u|$.

The following table summarizes the results of this section.

\begin{tabular}{|l|l|}
\hline \hline Problem & Time complexity \\
\hline Adjacency & $O\left(\left|A_{E}\right| \max (|u|,|v|)\right)$ \\
Existence of transient vertices & $O\left(\left|A_{E}\right|\right)$ \\
Existence of sinks, sources, isolated vertices & $O\left(2^{\left|A_{E}\right|}\right)$ \\
Finiteness of a vertex degree & $O\left(\left|A_{E}\right||v|\right)$ \\
Degree of a vertex & $O\left(2^{\left|A_{E}\right|}+\left|A_{E}\right||v|\right)$ \\
Infiniteness of a graph degree & $O\left(2^{\left|A_{E}\right|}\right)$ \\
\hline \hline
\end{tabular}

\section{Conclusions}

In this paper we have introduced a new way of specifying infinite hyper-graphs via regular languages. Our approach is similar to that used in [9] where the authors introduce a new representation of finite graphs. They use finite prefix-free languages of strings over alphabets which have themselves a graph structure. The strings of the language represent the vertices of the graph and there is an edge between two vertices if and only if the pair of the first two symbols, at which the two corresponding strings differ, is an edge in the alphabet. This approach applied to infinite graphs leads to a class of infinite graphs that either have an infinite degree (that is, there is a vertex with infinite edges incident on it) or are the disjoint union of infinitely many maximally connected subgraphs of finite size. Two interesting aspects in [9] are the use of prefix-free languages, which can be viewed as trees so that this approach presents the advantages of representing graphs by trees, and the relationships between graph operations and language operations (and then operations on the representation itself). Our aim was that of preserving these advantages also when infinite graphs are dealt with. We have also proved that the class of the equational graphs and the class of simple graphs introduced in [5] 
are strictly contained into the class of suffix-representable graphs. For the case of simple graphs, since the class of equational graphs is strictly contained in the simple graphs defined in [5], our result on the strict inclusion of the equational graphs could have been obtained via the strict inclusion of that class of graphs. However, our direct proof provides a way to obtain the representation of a given equational hyper-graph in the notation of suffix-representable graphs. We also introduce the class of infix-representable graphs and we discuss some properties and prove that it strictly includes the class of suffix-representable graphs.

Since the preliminary version of this paper appeared [13] some new results concerning this research have been published. In particular, it is worth mentioning the paper by Morvan [14] where he defines the class of rational graphs which can be characterized by binary relations recognized by rational transducers. This class turns out to be more general that the class of infix-representable graphs. In fact our class can be characterized by relations recognized by rational transducers with a bounded delay (that is the distance between the two heads of a 2-FA recognizing the corresponding relations cannot read, on the respective tapes, two locations which are arbitrarily far from each other). The results presented in Section 5.2 can be easily extended to this class of subgraphs.

As a future research we think that would be interesting to consider new classes of $f$-recognizable graphs. Moreover, introducing some constraints on $P$ and $l a b$, it is possible to determine some hierarchies whose investigation could give interesting hints on the use of this representation. Finally, we have considered some basic properties of graphs and sketched the algorithms to decide them on infixrepresentable graphs. It could be interesting, also, to consider in this class other problems, such as the existence of a path of a given length between any pair of vertices and, again, to determine the maximum size of a clique contained in the considered graph.

We would like to thank Didier Caucal for helpful discussions and suggestions.

\section{REFERENCES}

[1] S. Arnborg, J. Lagergren and D. Seese, Easy problems for tree-decomposable graphs. J. Algorithms 12 (1991) 308-340.

[2] K. Barthelmann, When Can an Equational Simple Graph Be Generated by Hyperedge Replacement?, in Proc. of the 23th International Symposium on Mathematical Foundations of Computer Science (MFCS'98), edited by L. Brim, J. Gruska and J. Zlatuska. Brno, Czech Republic, August 24-28, 1998, Lecture Notes in Comput. Sci. 1450 (1998) 543-552.

[3] M. Bauderon and B. Courcelle, Graph Expressions and Graph Rewritings. Math. Systems Theory 20 (1987) 83-127.

[4] H. L. Bodlaender and R. H. Möhring, The pathwidth and treewidth of cographs. SIAM J. Discrete Math. 6 (1993) 181-188.

[5] D. Caucal, On infinite transition graphs having a decidable monadic theory, in Proc. of 23rd International Colloquium on Automata, Languages and Programming (ICALP'96), edited by F.M. auf der Heide and B. Monien. Paderborn, Germany, July 8-12, 1996, Lecture Notes in Comput. Sci. 1099 (1996) 194-205. 
[6] D.G. Corneil, H. Lerchs and L. Stuart Burlingham, Complement reducible graphs. Discrete Appl. Math. 3 (1981) 163-174.

[7] B. Courcelle, The monadic second-order logic of graphs. II. Infinite graphs of bounded width. Mathematical Systems Theory 21 (1989) 187-221.

[8] B. Courcelle, The monadic second-order logic of graphs. III. Tree-width, forbidden minors and complexity issues. RAIRO: Theoret. Informatics Appl. 26 (1992) 257-286.

[9] A. Ehrenfeucht, J. Engelfriet and G. Rozenberg, Finite Languages for the Representation of Finite Graphs. J. Comput. System Sci. 52 (1996) 170-184.

[10] J. Engelfriet, T. Harju, A. Proskurowski and G. Rozenberg, Characterization and Complexity of Uniformly Non-primitive Labeled 2-Structures. Theoretical Comput. Sci. 154 (1996) 247-282.

[11] P.C. Fisher, Multi-Tape and Infinite-State Automata - A Survey. Comm. ACM 8 (1965) 799-805.

[12] J. Hopcroft and J. Ullman, Introduction to Automata Theory, Formal Languages and Computation. Addison-Wesley Publishing Company, Addison-Wesley Series in Comput. Sci. (1979)

[13] S. La Torre and M. Napoli, Representing Hyper-Graphs by Regular Languages. in Proc. of the 23th International Symposium on Mathematical Foundations of Computer Science (MFCS'98), edited by L. Brim, J. Gruska and J. Zlatuska, Brno, Czech Republic, August 24-28, 1998, Lecture Notes in Comput. Sci. 1450 (1998) 571-579.

[14] C. Morvan, On Rational Graphs, in Proc. of the 3rd International Conference on Foundations of Software Science and Computation Structures (FOSSACS 2000). Berlin, Germany, March 25 - April 2, 2000, Lecture Notes in Comput. Sci. 1784 (2000) 252-266.

[15] N. Robertson and P. Seymour, Graph Minors. II Algorithmic aspects of tree-width. J. Algorithms 7 (1986) 309-322.

[16] J. Valdez, R. E. Tarjan and E. Lawler, The recognition of series parallel digraphs. SIAM J. Comput. 11 (1982) 298-313.

Communicated by J. Gruska.

Received July, 1999. Accepted August, 2001. 